\title{
Clinical Application Study of Semi-cylindrical Beam Spoiler for Radiation Treatment of Early-stage Glottic Cancer Patients
}

\author{
DONG HYEOK CHOI ${ }^{1}$, SO HYUN AHN ${ }^{2 *}$, WOO SANG AHN ${ }^{3 *}$, SEONG SOO SHIN ${ }^{3}$ and JIN SUNG KIM ${ }^{2}$ \\ ${ }^{1}$ Department of Medicine, Yonsei University, Seoul, Republic of Korea; \\ ${ }^{2}$ Department of Radiation Oncology, Yonsei University College of Medicine, Seoul, Republic of Korea; \\ ${ }^{3}$ Department of Radiation Oncology, Gangneung Asan Hospital, \\ University of Ulsan College of Medicine, Gangneung, Republic of Korea
}

\begin{abstract}
Background/Aim: The purpose of this study was to determine whether a semi-cylindrical beam spoiler ( $s C B S$ ) developed herein effectively increases the skin dose in patients with early-stage glottic cancer. Patients and Methods: We measured the surface doses for 26 patients who used the sCBS during treatment of early-stage glottic cancer through a parallel-opposed lateral two-field $6 \mathrm{MV}$ photon beam. Measurements were performed by attaching optically stimulated luminescent dosimeters to the left, right, anterior (in-field), inferior, and superior (out-field) sides of the patient. Results: The measured results were $81.8 \pm 2.1 \%$ (left), $81.0 \pm 1.7 \%$ (right), and $76.8 \pm 2.7 \%$ (anterior) in the in-field region compared to prescription doses, with $5.7 \pm 1.7 \%$ (superior) and $2.7 \pm 0.7 \%$ (inferior) in the out-field region. Conclusion: sCBS can deliver a suitably ideal surface dose for treatment of early-stage glottic cancer.
\end{abstract}

Several studies have reported that the average 5-year overall survival rate for T1 glottic cancer is $85-95 \%$ (1-4). Thus, the survival rate for patients with early-stage glottic cancer is high; however, there are factors that affect local control, such as fraction size, biologically effective dose (BED) of a tumor, and inclusion/exclusion of the anterior commissure; many

This article is freely accessible online.

*These Authors contributed equally to this study.

Correspondence to: Woo Sang Ahn, Department of Radiation Oncology, Gangneung Asan Hospital, University of Ulsan College of Medicine, Gangneung 25440, Republic of Korea. Tel: +82 336105315, e-mail: anidol@ulsan.ac.kr; So Hyun Ahn, Department of Radiation Oncology, Yonsei University College of Medicine, Seoul 03722, Republic of Korea. Tel: +82 222284390, e-mail: mpsohyun@gmail.com

Key Words: Beam spoiler, glottic cancer, photon beam, surface dose, radiotherapy, optically stimulated luminescent dosimeter. studies have been conducted and reported on these factors (59). Kitani et al. evaluated prognostic factors in patients who received definitive radiation therapy from 1999 to 2011 and confirmed that the anterior commissure involvement was an important factor affecting local control (5). Lim et al. analyzed local failure in 222 patients with T1-2N0 squamouscell carcinoma of the glottis larynx who received definitive radiation therapy (RT) from 1981 to 2010 (6).

Therefore, during RT, it is necessary to ensure that the prescribed dose is sufficiently delivered to the entire lesion, including the anterior commissure located at a shallow depth. As noted in our previous work, bolus or beam spoilers for total body irradiation (TBI) can be considered as suitable options, but they are imperfect solutions owing to several shortcomings. In general, a bolus is used to increase the dose delivered to the skin area by compensating for insufficient electron balance when using megavolt treatment beams. However, since the head and neck regions have many curvatures due to anatomical characteristics, if a typical bolus is used, it may not be in close contact with the target area, and an air layer may be formed. On anatomically curved surfaces, air gaps are likely to occur between the bolus and skin, resulting in an underdose owing to the unexpected air gap. Butson et al. reported that an underdose of up to $6 \%$ may occur in the skin region from a $1 \mathrm{~cm}$ air gap (10). In addition, there may be difficulties in securing reproducibility during treatments that are repeated daily. The beam spoiler for TBI cannot be positioned close to the skin because of its structural characteristics; since the treatment for glottic cancer uses two bilateral beams, it may be cumbersome to move the beam spoiler for each field, and the position reproducibility with the beam spoiler may be poor.

Hence, we developed a dedicated beam spoiler for head and neck irradiation, called a semi-cylindrical beam spoiler (sCBS), to overcome these concerns and studied the percent depth dose (PDD) measurement and phantom treatment plan (11). Then, we applied this beam spoiler to actual patient treatments and measured the skin doses to check for accurate delivery of the 
Table I. Characteristics of the 26 glottic cancer patients: sex, age, location, grade, stage, ECOG performance, and commissure involvement for each patient.

\begin{tabular}{|c|c|c|c|c|c|c|c|}
\hline Patients & Gender & Age & Location & Grade & Stage & $\begin{array}{c}\text { ECOG } \\
\text { performance }\end{array}$ & $\begin{array}{l}\text { Commissure } \\
\text { involvement }\end{array}$ \\
\hline 1 & M & 78 & $\mathrm{Lt}$ & Low & $\mathrm{T} 1$ & 1 & \\
\hline 2 & M & 67 & $\mathrm{Lt}$ & Low & $\mathrm{T} 2$ & 1 & \\
\hline 3 & $\mathrm{M}$ & 66 & $\mathrm{Lt}$ & Low & $\mathrm{T} 1$ & 1 & \\
\hline 4 & M & 61 & $\mathrm{Lt}$ & Low & $\mathrm{T} 1$ & 1 & \\
\hline 5 & M & 71 & Rt & Low & $\mathrm{T} 1$ & 0 & \\
\hline 6 & M & 66 & Lt & Low & $\mathrm{T} 1$ & 1 & \\
\hline 7 & M & 72 & Both & Low & $\mathrm{T} 2$ & 1 & $\mathrm{Y}$ \\
\hline 8 & M & 84 & $\mathrm{Lt}$ & Low & $\mathrm{T} 1$ & 1 & \\
\hline 9 & M & 77 & Lt & Intermediate & $\mathrm{T} 1$ & 1 & \\
\hline 10 & M & 75 & Rt & Low & $\mathrm{T} 1$ & 1 & \\
\hline 11 & M & 66 & Rt & Low & $\mathrm{T} 1$ & 1 & $\mathrm{Y}$ \\
\hline 12 & M & 65 & Rt & Low & $\mathrm{T} 1$ & 1 & \\
\hline 13 & M & 79 & Lt & Low & $\mathrm{T} 1$ & 1 & \\
\hline 14 & M & 71 & Lt & Intermediate & $\mathrm{T} 2$ & 1 & \\
\hline 15 & M & 72 & Rt & Low & CIS & 1 & \\
\hline 16 & M & 82 & Rt & Low & $\mathrm{T} 1$ & 1 & \\
\hline 17 & M & 61 & Rt & Low & $\mathrm{T} 1$ & 1 & \\
\hline 18 & M & 67 & Rt & Intermediate & $\mathrm{T} 1$ & 1 & \\
\hline 19 & M & 65 & $\mathrm{Rt}$ & Low & $\mathrm{T} 1$ & 1 & \\
\hline 20 & M & 51 & Both & Low & $\mathrm{T} 2$ & 1 & \\
\hline 21 & M & 69 & $\mathrm{Lt}$ & Intermediate & $\mathrm{T} 1$ & 1 & \\
\hline 22 & M & 88 & Lt & Intermediate & $\mathrm{T} 1$ & 1 & \\
\hline 23 & M & 67 & $\mathrm{Lt}$ & Low & $\mathrm{T} 2$ & 1 & \\
\hline 24 & M & 67 & Rt & Low & $\mathrm{T} 1$ & 1 & \\
\hline 25 & M & 71 & Lt & Low & $\mathrm{T} 2$ & 1 & \\
\hline 26 & M & 80 & Rt & Low & $\mathrm{T} 1$ & 1 & \\
\hline
\end{tabular}

M: Male.

prescribed dosage. The reason for using the device in actual treatment is that unlike a phantom, the body weight of a patient may change over the course of the treatment, and there may be daily variations in the setup. Since the treatment is $2 \mathrm{D}$ in nature and does not use image guided radiation therapy (IGRT), the reproducibility was confirmed through in vivo dosimetry.

\section{Patients and Methods}

Semi-cylindrical beam spoiler ( $s C B S$ ). We used an sCBS for earlystage glottic cancer RT along with a parallel-opposed lateral two-field $6 \mathrm{MV}$ photon beam. The sCBS was made of polymethylmethacrylate (PMMA, density: $1.18 \mathrm{~g} / \mathrm{cm}^{3}$ ) and had an inner radius of $18 \mathrm{~cm}$ and a thickness of $0.5 \mathrm{~cm}$ with respect to the semi-cylindrical shape (11).

Patients. A total of 26 early-stage glottic cancer patients who were treated with the sCBS from February 2012 to March 2016 were enrolled in this study. The details regarding the patients are summarized in Table I. All patients were treated with two-opposing, bilateral beams using a Varian Clinac iX (Varian Medical Systems, Palo Alto, CA, USA). In addition, the sCBS was applied to the patients during computed tomography (CT) simulation and treatment. Figure 1 shows the CT images of a patient with the sCBS. The source-to-surface distance (SSD) was set to $100 \mathrm{~cm}$, and the dose rate and field size were set to $500 \mathrm{MU} / \mathrm{s}$ and $10 \times 10 \mathrm{~cm}^{2}$,
A

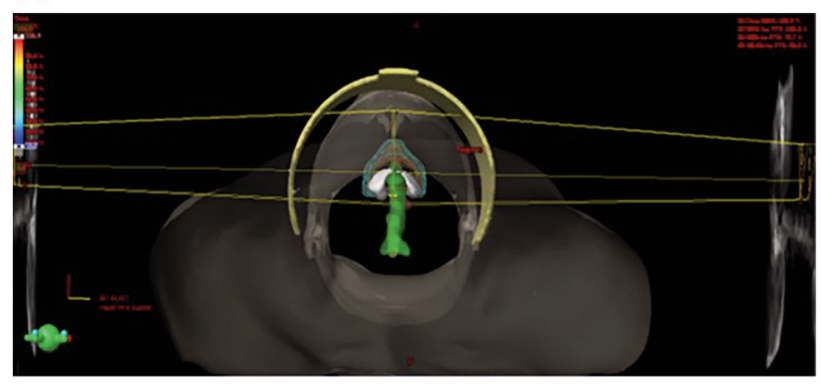

B

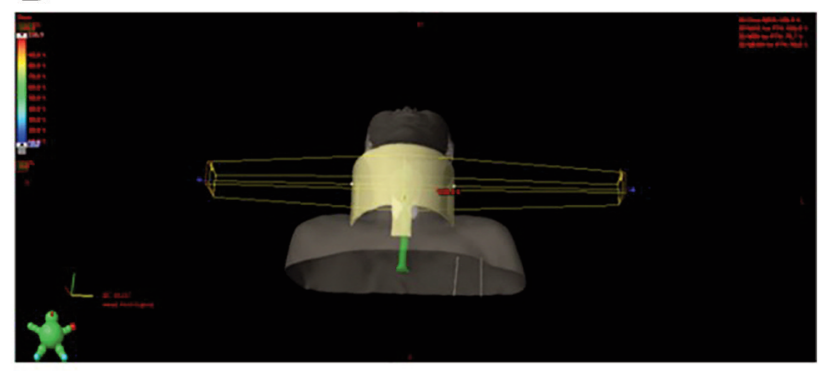

Figure 1. Computed tomography simulation of a patient with a semicylindrical beam spoiler applied: (A) cross-sectional view; (B) front view. 
Table II. Surface dose to the patients as measured through optically stimulated luminescent dosimeters: left, right, and anterior surface doses measured in the in-field condition, and external dose on the superior and inferior regions as measured in the out-field condition and ratio of received to prescribed dose.

\begin{tabular}{|c|c|c|c|c|c|c|c|c|c|c|c|}
\hline \multirow{3}{*}{ Patients } & \multirow{3}{*}{$\begin{array}{c}\text { Number of } \\
\text { measurements }\end{array}$} & \multicolumn{6}{|c|}{ In-field (surface dose) } & \multicolumn{4}{|c|}{ Out-field (external dose) } \\
\hline & & \multicolumn{2}{|c|}{ Left side } & \multicolumn{2}{|c|}{ Right side } & \multicolumn{2}{|c|}{ Anterior } & \multicolumn{2}{|c|}{ Superior } & \multicolumn{2}{|c|}{ Inferior } \\
\hline & & cGy & $\%$ & cGy & $\%$ & cGy & $\%$ & cGy & $\%$ & cGy & $\%$ \\
\hline 1 & 2 & $156.5 \pm 0.6$ & $(78.3 \pm 0.3)$ & $155.3 \pm 4.1$ & $(77.6 \pm 2.0)$ & $154.6 \pm 2.3$ & $(77.3 \pm 1.2)$ & - & - & - & - \\
\hline 2 & 3 & $165.1 \pm 13.0$ & $(82.5 \pm 6.5)$ & $166.0 \pm 4.3$ & $(83.0 \pm 2.1)$ & $169.8 \pm 9.9$ & $(84.9 \pm 5.0)$ & - & - & - & - \\
\hline 3 & 3 & $168.8 \pm 3.0$ & $(84.4 \pm 1.5)$ & $165.3 \pm 3.6$ & $(82.2 \pm 1.4)$ & $164.2 \pm 1.2$ & $(82.7 \pm 1.5)$ & - & - & - & - \\
\hline 4 & 4 & $158.8 \pm 2.3$ & $(79.4 \pm 1.2)$ & $157.3 \pm 3.9$ & $(78.6 \pm 2.0)$ & $161.4 \pm 2.5$ & $(80.7 \pm 1.3)$ & - & - & - & - \\
\hline 5 & 4 & $168.1 \pm 1.8$ & $(84.0 \pm 0.9)$ & $165.2 \pm 3.0$ & $(82.7 \pm 1.5)$ & $164.5 \pm 2.9$ & $(82.2 \pm 1.4)$ & - & - & - & - \\
\hline 6 & 5 & $163.7 \pm 5.3$ & $(81.9 \pm 3.1)$ & $163.8 \pm 6.1$ & $(81.8 \pm 2.6)$ & $131.8 \pm 16.4$ & $(65.9 \pm 8.2)$ & - & - & - & - \\
\hline 7 & 2 & $158.7 \pm 6.5$ & $(79.4 \pm 3.2)$ & $157.5 \pm 3.6$ & $(78.8 \pm 1.8)$ & $141.3 \pm 2.4$ & $(70.7 \pm 1.2)$ & $10.2 \pm 8.9$ & $(5.1 \pm 4.5)$ & - & - \\
\hline 8 & 1 & 153.3 & 76.6 & 154.9 & 76.5 & 156.3 & 78.2 & 4.5 & 2.3 & 2.7 & 1.3 \\
\hline 9 & 1 & 156.9 & 78.5 & 153.5 & 76.8 & 145.8 & 72.9 & 4.2 & 2.1 & 3.0 & 1.5 \\
\hline 10 & 1 & 164.5 & 82.3 & 166.1 & 83 & 142.7 & 71.3 & 6.4 & 3.2 & 3.4 & 1.7 \\
\hline 11 & 2 & $175.5 \pm 0.3$ & $(87.7 \pm 0.2)$ & $171.2 \pm 1.3$ & $(85.6 \pm 0.7)$ & $160.4 \pm 1.8$ & $(80.2 \pm 0.9)$ & $10.9 \pm 6.1$ & $(5.5 \pm 3.1)$ & $2.8 \pm 0.7$ & $(1.4 \pm 0.4)$ \\
\hline 12 & 2 & $170.3 \pm 0.6$ & $(85.2 \pm 0.3)$ & $160.0 \pm 0.8$ & $(80.0 \pm 0.4)$ & $153.7 \pm 3.1$ & $(76.9 \pm 1.6)$ & $4.7 \pm 4.7$ & $(2.4 \pm 2.3)$ & $5.2 \pm 5.4$ & $(2.6 \pm 2.7)$ \\
\hline 13 & 2 & $154.9 \pm 5.5$ & $(77.4 \pm 2.7)$ & $152.9 \pm 5.4$ & $(76.5 \pm 2.7)$ & $137.6 \pm 7.5$ & $(68.8 \pm 3.7)$ & $2.7 \pm 0.4$ & $(1.4 \pm 0.2)$ & $1.8 \pm 0.8$ & $(0.9 \pm 0.4)$ \\
\hline 14 & 3 & $163.2 \pm 5.4$ & $(81.6 \pm 2.7)$ & $170.9 \pm 0.4$ & $(85.4 \pm 0.2)$ & $169.2 \pm 0.2$ & $(84.6 \pm 0.1)$ & $2.3 \pm 0.8$ & $(1.1 \pm 0.4)$ & $1.3 \pm 0.2$ & $(0.7 \pm 0.1)$ \\
\hline 15 & 3 & $177.5 \pm 2.4$ & $(88.7 \pm 1.2)$ & $167.0 \pm 3.9$ & $(83.5 \pm 2.0)$ & $165.7 \pm 4.7$ & $(82.9 \pm 2.3)$ & $1.6 \pm 1.7$ & $(0.8 \pm 0.9)$ & $2.1 \pm 1.7$ & $(1.0 \pm 0.9)$ \\
\hline 16 & 3 & $172.4 \pm 5.3$ & $(86.2 \pm 2.6)$ & $166.0 \pm 0.1$ & $(83.0 \pm 0.1)$ & $162.2 \pm 2.6$ & $(81.1 \pm 1.3)$ & $1.9 \pm 0.2$ & $(1.0 \pm 0.1)$ & $0.7 \pm 0.0$ & $(0.3 \pm 0.0)$ \\
\hline 17 & 3 & $169.4 \pm 3.7$ & $(84.7 \pm 1.8)$ & $163.0 \pm 4.6$ & $(81.5 \pm 2.3)$ & $157.0 \pm 6.2$ & $(78.5 \pm 3.1)$ & $8.0 \pm 3.7$ & $(4.0 \pm 1.8)$ & $3.4 \pm 0.5$ & $(1.7 \pm 0.2)$ \\
\hline 18 & 3 & $168.1 \pm 1.1$ & $(84.1 \pm 0.6)$ & $159.6 \pm 0.9$ & $(79.8 \pm 0.5)$ & $149.4 \pm 4.9$ & $(74.7 \pm 2.4)$ & $6.2 \pm 6.1$ & $(3.1 \pm 3.1)$ & $2.4 \pm 1.0$ & $(1.2 \pm 0.5)$ \\
\hline 19 & 3 & $161.9 \pm 8.4$ & $(81.0 \pm 4.2)$ & $157.2 \pm 7.8$ & $(78.6 \pm 3.9)$ & $148.9 \pm 9.8$ & $(74.5 \pm 4.9)$ & $5.1 \pm 3.0$ & $(2.6 \pm 1.5)$ & $2.1 \pm 0.5$ & $(1.1 \pm 0.2)$ \\
\hline 20 & 3 & $157.5 \pm 3.3$ & $(78.8 \pm 1.6)$ & $160.1 \pm 4.7$ & $(80.1 \pm 2.4)$ & $154.6 \pm 9.8$ & $(77.3 \pm 4.9)$ & $7.6 \pm 5.9$ & $(3.8 \pm 3.0)$ & $4.6 \pm 2.8$ & $(2.3 \pm 1.4)$ \\
\hline 21 & 4 & $165.6 \pm 2.4$ & $(82.8 \pm 1.2)$ & $162.3 \pm 4.5$ & $(81.2 \pm 2.2)$ & $149.0 \pm 3.4$ & $(74.5 \pm 1.7)$ & $5.0 \pm 2.8$ & $(2.6 \pm 1.5)$ & $1.5 \pm 0.4$ & $(1.1 \pm 0.2)$ \\
\hline 22 & 4 & $158.8 \pm 1.7$ & $(79.4 \pm 0.9)$ & $165.8 \pm 2.4$ & $(82.9 \pm 1.2)$ & $153.2 \pm 2.3$ & $(76.6 \pm 1.2)$ & $6.7 \pm 4.2$ & $(3.4 \pm 2.1)$ & $2.3 \pm 2.2$ & $(1.1 \pm 1.1)$ \\
\hline 23 & 4 & $158.4 \pm 8.9$ & $(79.2 \pm 4.5)$ & $161.0 \pm 5.7$ & $(80.5 \pm 2.9)$ & $144.5 \pm 14.2$ & $(72.3 \pm 7.1)$ & $7.0 \pm 4.2$ & $(3.5 \pm 2.1)$ & $2.5 \pm 2.0$ & $(1.2 \pm 1.0)$ \\
\hline 24 & 4 & $165.9 \pm 5.3$ & $(82.9 \pm 2.6)$ & $167.8 \pm 1.7$ & $(83.9 \pm 0.8)$ & $150.8 \pm 3.3$ & $(75.4 \pm 1.6)$ & $7.7 \pm 3.6$ & $(3.9 \pm 1.8)$ & $4.5 \pm 4.2$ & $(2.3 \pm 2.1)$ \\
\hline 25 & 4 & $154.3 \pm 4.4$ & $(77.1 \pm 2.2)$ & $163.6 \pm 3.2$ & $(81.8 \pm 1.6)$ & $148.2 \pm 5.7$ & $(74.1 \pm 2.9)$ & $3.5 \pm 0.3$ & $(1.7 \pm 0.1)$ & $3.1 \pm 1.1$ & $(1.5 \pm 0.5)$ \\
\hline \multirow[t]{4}{*}{26} & 5 & $167.3 \pm 3.0$ & $(83.6 \pm 1.5)$ & $162.9 \pm 3.2$ & $(81.5 \pm 1.6)$ & $154.0 \pm 4.1$ & $(77.0 \pm 2.1)$ & $6.8 \pm 2.0$ & $(3.4 \pm 1.0)$ & $1.8 \pm 0.9$ & $(1.7 \pm 0.2)$ \\
\hline & Average & $163.7 \pm 4.1$ & $81.8 \pm 2.1$ & $162.2 \pm 3.4$ & $81.0 \pm 1.7$ & $153.5 \pm 5.3$ & $76.8 \pm 2.7$ & $5.7 \pm 3.4$ & $2.8 \pm 1.7$ & $2.7 \pm 1.5$ & $1.4 \pm 0.7$ \\
\hline & MIN & 153.3 & 76.6 & 152.9 & 76.5 & 131.8 & 65.9 & 1.6 & 0.8 & 0.7 & 0.3 \\
\hline & MAX & 177.5 & 88.7 & 171.2 & 85.6 & 169.8 & 84.9 & 10.9 & 5.5 & 5.2 & 2.6 \\
\hline
\end{tabular}

respectively. The $6 \mathrm{MV}$ photon beam was then used to deliver the prescribed dose of $200 \mathrm{cGy}$.

Measurements. As shown in Figure 2, optically stimulated luminescent dosimeters (OSLDs) were attached to the left side, right side, anterior (in-field) direction, inferior direction, and superior (out-field) direction to measure the surface dose. The number of measurements performed was 1-5 fractions per patient, and the ratio of surface dose according to the prescribed dose was obtained. Our study was based on at least three in vivo measurements per patient. However, if repeated measurements were impossible owing to the conditions of the treatment room, only one or two in vivo measurements were obtained. Furthermore, if the in vivo results measured thrice had large deviations, up to five measurements were performed.

\section{Results}

Table II shows the surface dose for each patient and ratio of surface dose per prescribed dose (200 cGy). The average in-
A

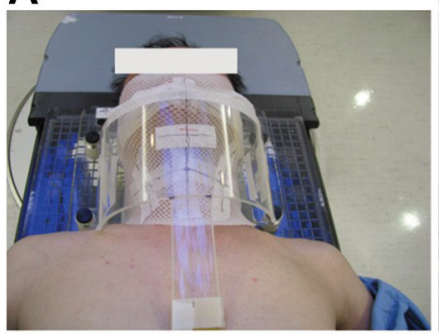

B

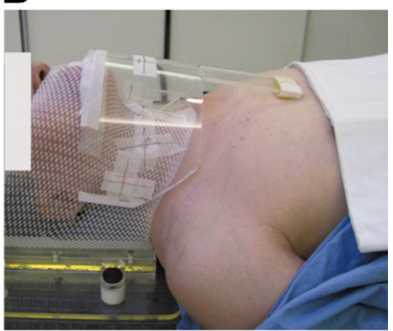

Figure 2. Optically stimulated luminescent dosimeters attached to a patient with a semi-cylindrical beam spoiler applied: (A) front view; (B) side view.

field surface doses obtained were 163.7 $\pm 4.1(81.8 \pm 2.1 \%)$, $162.2 \pm 3.4(81.0 \pm 1.7 \%)$, and $153.5 \pm 5.3(76.8 \pm 2.7 \%) \mathrm{cGy}$ for the left side, right side, and anterior direction, respectively. The outfield measurement results were $5.7 \pm 1.7(2.8 \pm 1.7 \%)$ 
and $2.7 \pm 1.5(1.4 \pm 0.7 \%) \mathrm{cGy}$ in the superior and inferior directions, respectively. The average measured deviation by fraction was $2.2 \%$, and the largest deviation was $8.2 \%$.

\section{Discussion}

We conducted a study to evaluate the basic dose characteristics, such as PDD, using the previously developed sCBS with a phantom (11). From the results of the previous phantom study, it was confirmed that when the sCBS was applied, a dose of about $85.13 \%$ of the prescribed dose entered the skin. From the measured results in patients in this study, it was confirmed that the expected build-up effect was adequately exhibited, with $81.8 \%$ and $81.0 \%$ delivered doses for the left and right sides, respectively.

In principle, we decided to measure more than three fractions to increase the reliability of the measurements. However, there were patients from whom measurements could not be obtained owing to their treatment times, therefore, only one or two measurements were recorded. In addition, there were patients from whom four or five measurements were obtained owing to large differences in the first three measurements. As shown in Table II, it is observed that there is a deviation of up to $8.2 \%$ during repeated measurements, which is attributed to patient movements during the treatment, weight variations, or changes in the measurement positions.

As shown in the results, the measured result from the anterior direction is lower than those from the left and right sides, which is attributed to underestimations due to the influence of angular dependence because the angle between the active window of the OSLDs and beam incident direction is $90^{\circ}(12-14)$. The sCBS developed exclusively for head and neck treatments in this study need not be moved from one field to the next during treatment, does not need customization for each patient, and has the advantage of a simple setup. However, if IMRT or VMAT treatments are performed, the beam spoiler's positional reproducibility must be more precise than that available at present through IGRT because IMRT is more sensitive to positional accuracy by transmitting beams using small beamlets using multi leaf collimator (MLC) (15-19).

\section{Conclusion}

This study explored the clinical application and usefulness of the sCBS through evaluation of the surface dose accumulation characteristics during treatment for early-stage glottic cancer. The sCBS provides improved build-up to the beam, thereby providing increased skin protection and helping deliver a sufficient percentage of the prescribed dose. In addition, it is very convenient for clinical use in the field, with a simple installation method. Therefore, we confirm that the sCBS is a useful tool for early-stage glottic cancer RT.

\section{Conflicts of Interest}

The Authors declare no conflicts of interest in relation to this study.

\section{Authors' Contributions}

Conceptualization: DHC and WSA; methodology: WSA and SA; data curation: WSA; formal analysis: DHC; funding acquisition: WSA and SA, DHC and WSA; supervision: WSA and SA; validation: JSK and SSS; visualization: JSK and SSS; writingoriginal draft: DHC and SA; writing-review and editing: WSA, DHC, and SA.

\section{Acknowledgements}

This research was supported by the Nuclear Safety Research Program through the Korea Foundation of Nuclear Safety (KoFONS) using financial resources granted by the Nuclear Safety and Security Commission (NSSC) of the Republic of Korea (No. 2003013), National Research Foundation of Korea (NRF) grant funded by the Korean government (MSIT) (No. 2019R1A2C108912912), Basic Science Research Program through the National Research Foundation of Korea (NRF) grant funded by the Ministry of Education (Nos. 2018R1D1A1B07050217 and 2021R1I1A1A01057995), and Medical Research Promotion Program through the Gangneung Asan Hospital funded by the Asan Foundation (No. 2021IC003).

\section{References}

1 Ambrosch P and Fazel A: Functional organ preservation in laryngeal and hypopharyngeal cancer. GMS Curr Top Otorhinolaryngol Head Neck Surg 10: Doc02, 2011. PMID: 22558052. DOI: $10.3205 /$ cto000075

2 Mendenhall WM, Amdur RJ, Morris CG and Hinerman RW: T1T2N0 squamous cell carcinoma of the glottic larynx treated with radiation therapy. J Clin Oncol 19(20): 4029-4036, 2001. PMID: 11600604. DOI: 10.1200/JCO.2001.19.20.4029

3 Johansen LV, Grau C and Overgaard J: Glottic carcinomapatterns of failure and salvage treatment after curative radiotherapy in 861 consecutive patients. Radiother Oncol 63(3): 257-267, 2002. PMID: 12142089. DOI: 10.1016/s01678140(02)00118-4

4 Chera BS, Amdur RJ, Morris CG, Kirwan JM and Mendenhall WM: T1N0 to T2N0 squamous cell carcinoma of the glottic larynx treated with definitive radiotherapy. Int J Radiat Oncol Biol Phys 78(2): 461-466, 2010. PMID: 20153124. DOI: 10.1016/j.ijrobp.2009.08.066

5 Kitani Y, Kubota A, Furukawa M and Sato K: Prognostic factors for local control in patients receiving radiation therapy for early glottic cancer: anterior commissure involvement and effect of chemoradiotherapy. Eur Arch Otorhinolaryngol 273(4): 10111017, 2016. PMID: 25726168. DOI: 10.1007/s00405-015-3579-8

6 Lim YJ, Wu HG, Kwon TK, Hah JH, Sung MW, Kim KH and Park CI: Long-term outcome of definitive radiotherapy for early glottic cancer: Prognostic factors and patterns of local failure. Cancer Res Treat 47(4): 862-870, 2015. PMID: 25687859. DOI: 10.4143/crt.2014.203

7 Tulli M, Re M, Bondi S, Ferrante L, Dajko M, Giordano L, Gioacchini FM, Galli A and Bussi M: The prognostic value of 
anterior commissure involvement in T1 glottic cancer: A systematic review and meta-analysis. Laryngoscope 130(8): 1932-1940, 2020. PMID: 31721216. DOI: 10.1002/lary.28395

8 Hendriksma M and Sjögren EV: Involvement of the anterior commissure in early glottic cancer (Tis-T2): a review of the literature. Cancers (Basel) 11(9): 1234, 2019. PMID: 31450795. DOI: $10.3390 /$ cancers 11091234

9 Tong CC, Au KH, Ngan RK, Chow SM, Cheung FY, Fu YT, Au JS and Law SC: Impact and relationship of anterior commissure and time-dose factor on the local control of T1N0 glottic cancer treated by $6 \mathrm{MV}$ photons. Radiat Oncol 6: 53, 2011. PMID: 21600025. DOI: $10.1186 / 1748-717 X-6-53$

10 Butson MJ, Cheung T, Yu P and Metcalfe P: Effects on skin dose from unwanted air gaps under bolus in photon beam radiotherapy. Radiat Measurements 32(3): 201-204, 2000.

11 Kerns JR, Kry SF, Sahoo N, Followill DS and Ibbott GS: Angular dependence of the nanoDot OSL dosimeter. Med Phys 38(7): 3955-3962, 2011. PMID: 21858992. DOI: 10.1118/1.3596533

12 Shin SS, Ahn S, Baek SH, Kwak J, Kim JS and Ahn WS: Usefulness of semi-cylindrical beam spoiler in treatment of early glottic cancer using $6 \mathrm{MV}$ photon beam. In Vivo 36(1): 465-472, 2022. PMID: 34972750. DOI: 10.21873/invivo.12726

13 Lehmann J, Dunn L, Lye JE, Kenny JW, Alves AD, Cole A, Asena A, Kron T and Williams IM: Angular dependence of the response of the nanoDot OSLD system for measurements at depth in clinical megavoltage beams. Med Phys 41(6): 061712, 2014. PMID: 24877807. DOI: 10.1118/1.4875698

14 Jursinic PA: Angular dependence of dose sensitivity of nanoDot optically stimulated luminescent dosimeters in different radiation geometries. Med Phys 42(10): 5633-5641, 2015. PMID: 26429237. DOI: $10.1118 / 1.4929558$

15 Schwartz DL, Garden AS, Shah SJ, Chronowski G, Sejpal S, Rosenthal DI, Chen Y, Zhang Y, Zhang L, Wong PF, Garcia JA, Kian Ang K and Dong L: Adaptive radiotherapy for head and neck cancer-dosimetric results from a prospective clinical trial. Radiother Oncol 106(1): 80-84, 2013. PMID: 23369744. DOI: 10.1016/j.radonc.2012.10.010
16 Rock K, Huang SH, Tiong A, Lu L, Xu W, Ringash J, Bratman SV, Tong L, Chan B, Cho J, Giuliani M, Hope A, Bayley A, Kim J, de Almeida JR, O'Sullivan B and Waldron J: Partial laryngeal IMRT for T2N0 glottic cancer: Impact of image guidance and radiation therapy intensification. Int J Radiat Oncol Biol Phys 102(4): 941-949, 2018. PMID: 29730062. DOI: 10.1016/j.ijrobp. 2018.03.034

17 van Kranen S, van Beek S, Rasch C, van Herk M and Sonke JJ: Setup uncertainties of anatomical sub-regions in head-and-neck cancer patients after offline CBCT guidance. Int J Radiat Oncol Biol Phys 73(5): 1566-1573, 2009. PMID: 19306753. DOI: 10.1016/j.ijrobp.2008.11.035

18 Ove R, Cavalieri R, Noble D and Russo SM: Variation of neck position with image-guided radiotherapy for head and neck cancer. Am J Clin Oncol 35(1): 1-5, 2012. PMID: 21278564. DOI: $10.1097 / \mathrm{COC} .0 \mathrm{~b} 013 \mathrm{e} 3181 \mathrm{fe} 46 \mathrm{bb}$

19 Neubauer E, Dong L, Followill DS, Garden AS, Court LE, White RA and Kry SF: Assessment of shoulder position variation and its impact on IMRT and VMAT doses for head and neck cancer. Radiat Oncol 7: 19, 2012. PMID: 22316381. DOI: 10.1186/1748-717X-7-19 